\title{
Effectiveness of one-to-one peer support for patients with severe mental illness - a randomised controlled trial
}

\author{
C. I. Mahlkea, S. Priebe ${ }^{b}$, K. Heumann ${ }^{a}$, A. Daubmann', K. Wegscheiderc, T. Bock ${ }^{\text {a }}$ \\ a University Medical Center Hamburg Eppendorf, Center for Psychosocial Medicine, Martinistr. 52, 20249 Hamburg, Germany \\ ${ }^{b}$ Queen Mary University of London, Unit for Social and Community Psychiatry (WHO Collaborating Centre for Mental Health \\ Service Development), Newham Centre for Mental Health, London E13 8SP, United Kingdom \\ c University Medical Center Hamburg Eppendorf, Department of Medical Biometry and Epidemiology, Martinistr. 52, 20249 \\ Hamburg, Germany
}

\section{Corresponding Author}

Dipl.Psych. Candelaria I. Mahlke

University Medical Center Hamburg Eppendorf

Martinistr. 52, 20246 Hamburg

Tel.: ++49 (0)40 741058933

Fax: ++49 (0)40 7410555455

c.mahlke@uke.de

\begin{abstract}
\section{Background}

One-to-one peer support is a resource-oriented approach for patients with severe mental illness. Existing trials provided inconsistent results and commonly have methodological shortcomings, such as poor training and role definition of peer supporters, small sample sizes, and lack of blinded outcome assessments.

\section{Methods}

This is a randomised controlled trial comparing one-to-one peer support with treatment as usual. Eligible were patients with severe mental illnesses: psychosis, major depression, bipolar disorder or borderline personality disorder of more than two years' duration. A total of 216 patients were recruited through in- and out-patient services from four hospitals in Hamburg, Germany, with 114 allocated to the intervention group and 102 to the control group. The intervention was one-to-one peer support, delivered by trained peers and according to a defined role specification, in addition to treatment as usual over the course of six months, as compared to treatment as usual alone.

Primary outcome was self-efficacy measured on the General Self-Efficacy Scale at six-month follow up. Secondary outcomes included quality of life, social functioning, and hospitalisations.

\section{Results}

Patients in the intervention group had significantly higher scores of self-efficacy at the sixmonth follow up. There were no statistically significant differences on secondary outcomes in the intention to treat analyses.

\section{Conclusions}

The findings suggest that one-to-one peer support delivered by trained peer supporters can improve self-efficacy of patients with severe mental disorders over a one-year period. Oneto-one peer support may be regarded as an effective intervention. Future research should explore the impact of improved self-efficacy on clinical and social outcomes.

\section{Funding}

This project was part of the 'psychenet' project (www.psychenet.de) and received funding from the Federal Ministry of Education and Research in Germany from 2011 until 2015 (BMBF-Nr: O1KQ1002B). The project had further study arms that employed qualitative methods as process evaluation that will be published separately. 


\section{Introduction}

The aims of mental health care of people with persistent and severe mental illnesses are no longer limited to aspects of clinical recovery, like symptom control and functioning. Care also aims to increase people's capacities for a largely independent lifestyle and comprehensive social and professional inclusion within the limitations caused by persistent symptoms, as it is captured in the personal recovery concept $[1,2]$. Flexible settings, with low service access thresholds are necessary to meet the individual, complex needs of this target group on their way to recovery.

\subsection{Peer support in mental health care}

One method to promote personal recovery is peer support of individuals who experienced mental health crisis and the path to recovery themselves. Peer support is a resourceoriented, instead of a deficit-oriented, approach and therefore not disorder specific [3]. It has its origins in informal and user-led care initiatives $[4,5]$. With a greater focus on recovery oriented and person-centred interventions in statutory mental health services $[1,6]$, there has been a shift to more formalised peer support models arranged and led by staff [7], which risks jeopardizing peer support's unique culture. Peer workers in mental health care have progressed in recovery from a mental illness themselves, to a stage where they can manage their illness, persistent symptoms, and pursue a fulfilling life [8, 9]. This experiential knowledge on the way towards recovery is a valuable resource in mental health service delivery and, when shared in self-disclosure with individuals in crisis, may contribute to their recovery by initiating a process that strengthens patients' self-efficacy beliefs $[9,10]$. Mead states that peer support helps to establish an alternative, more positive self-image that goes beyond a mere, passive patient identity [10].

\subsection{Change model of peer support}

Research on formal peer support has addressed the benefits for patients and peer supporters themselves, the challenges for institutions and peer supporters, and the factors facilitating successful implementation [9]. Anyway, the theoretical background of peer support is still underdeveloped. The first change model of peer support by Gillard is based on a comparative case study of ten peer worker initiatives in statutory and informal mental health services [11]. They conducted in-depth interviews with 71 peer workers, service users, staff, and managers. The author emphasises two parallel mechanisms promoting recovery: on the one hand the bridge into services that can be built by peer workers for individuals with severe mental health crises who have complex treatment needs but distrust in mental health services. Thus, implementing effective peer support in clinical practice, the protection of a peer support specific culture and a high level of flexibility in how peers support different patients have been suggested as essential $[7,11]$. Gillard et al. recommend using a whole system approach on recovery orientation, that protects the authenticity of peers' identity and practice, and that acknowledges that there is an impact on organisational culture, processes, and way of working [11]. Secondly, on the other hand the role-modelling of recovery by peer workers and its underpinning effect on the patients' self-efficacy beliefs [11]. High self-efficacy reflects ones' capability to tackle novel tasks, coping with adversity in a broad range of stressful life events or challenging encounters, and recover from setbacks more quickly [12].

\subsection{Effectiveness of peer support}

Reviews of effectiveness studies of peer support report inconsistent positive effects on different outcomes $[13,14,15,16]$. A systematic review of eleven randomised controlled trials [14] found significantly reduced emergency service use and a higher number of met needs in patients receiving peer support. Another review and meta-analysis [9] included five trials and identified a small positive effect on quality of life. Neither of the reviews suggested any effects on clinical outcomes or general functioning. However, all reviews criticise the 
limited quality of the included studies $[13,14,15,16]$. Shortcomings include poorly defined roles of the peer supporters, little training for peer supporters and mental health staff, small sample sizes, no blinded outcome assessments, and a lack of recovery-oriented outcome criteria.

We therefore conducted a trial testing the effectiveness of one-to-one peer support with defined roles and extensive training for peer supporters. We employed a structured preparation of mental health staff in participating services, and self-efficacy as a recoveryoriented primary outcome criterion.

\section{Material and Method}

We conducted a multi-site, parallel-arm, randomised controlled trial in Hamburg, Germany. This was a superiority trial, testing one-to-one peer support in addition to treatment as usual, as compared to treatment as usual alone.

\subsection{Participants}

Patients were recruited through in- and out-patient services from four psychiatric hospitals (University Medical Centre Hamburg-Eppendorf, Asklepios Clinic North, Albertinen Hospital, and Schön Clinic Hamburg Eilbek) in Hamburg.

We included patients meeting the definition of severe mental illness according to the German Association of Psychiatry, Psychotherapy and Neurology (DGPPN) [17]. Eligible patients were aged between $18-80$ years with a primary diagnosis of schizophrenia and related disorders (F2), affective disorders (F3), or personality disorder (F6) and a duration of illness of more than two years. Exclusion criteria were a primary diagnosis of drug or alcohol abuse and an insufficient command of German to communicate with the peer supporters.

\subsection{Randomisation}

The participants were randomly allocated to either one-to-one peer support or the control group in a 1:1 ratio, stratified by hospital, in blocks of 20. An independent statistician, working in the Department of Medical Biometry and Epidemiology of the University Medical Centre Hamburg-Eppendorf produced randomly generated treatment allocations using SAS Institute Inc., Cary, NC, USA, Version 9.3 within sealed, numbered, opaque envelopes that were stored and inaccessible to the trial team.

\subsection{Procedures}

Patients were recruited through in- and out-patient services. If still in hospital, the patient could make his first appointment with the peer supporter shortly before discharge, and continue as an outpatient. Interested individuals attended a first information appointment, where a researcher obtained written informed consent and conducted the baseline assessment, after which the patient was randomly allocated to one of the two groups.

Patients in both groups were contacted by a researcher again after 6 and 12 months, and were sent the set of self-rated questionnaires. At the same time points, the treating psychiatrists (either in an outpatient service of a hospital or in a private office practice) were also contacted and sent the relevant questionnaires for external assessment. If the questionnaires were not returned, a reminder was sent after one week. After two weeks, researchers tried to contact participants and treating psychiatrists by telephone for a further reminder.

\subsection{Intervention}

\subsubsection{Training of staff and peers}


In line with suggestions for best practice models $[18,19,20]$, structured training for peers and staff was implemented, involving peer supporters as trainers.

All peer supporters attended a structured training program based on the Ex-In Curriculum, which was delivered by a peer worker and a psychologist [21]. It took a total of 192 hours, arranged on 12 weekend workshops from Friday afternoon to Sunday afternoon. Training consists of five basic modules (1. Promoting Health, Wellness and Well-being 2. Empowerment in theory and practice 3. Experience and Participation 4. Trialogue 5. Perspectives and experiences of recovery) and four specialised modules (6. Independent Peer Advocacy in Mental Health 7. Recovery based assessment and planning for people in crisis 8 . Accepting and making sense of hearing voices and other persistent symptoms 9 . Peer Support). The training includes two practical units of two months duration each in mental health services.

For mental health staff, two research assistants and one peer worker from the given hospital facilitated two-hour workshops before the implementation of peer support, providing information on recovery orientation and the role and tasks of peer supporters.

\subsubsection{Supervision}

Once the intervention had started, biweekly group-supervision was provided by a psychologist and trainer from the peer-education organisation, Ex-In Hamburg. Further individual supervision was provided upon request. To protect the independence of peer supporters, they were not part of the clinical team in the service, but formed their own teams along with family peer workers at each site. Peer supporters were based in separate offices in the outpatient services area in the participating hospitals.

\subsubsection{Role description and tasks}

People working as a peer supporter were characterised by [21]:

- The ability to reflect their experiences and their coping

- The competence and the willingness to actively use their own experiences as a resource, to make it visible for users and to argue them critically

- The conscious use of their own experiences to reduce inhibitions or challenge appreciations and prejudices

- A constructive approach in dealing with public stigmatisation

The role of the peer supporters was clearly described in a job specification, but not further manualised in detail, to allow sufficient flexibility. The aim for peer supporters was to help patients advance in their recovery by enhancing a sense of control over their lives. They were supposed to bring in their own experiences and coping strategies, and work according to some basic values and assumptions: providing support without a hierarchy, accepting that nobody is only ill or only healthy, using crises as an opportunity for a next step, accepting and integrating the experience, and seeing peer support as a process without focusing on clinical outcomes. The specific tasks included practical support with daily life, helping to endure and understand crises, sharing ideas about individual planning and recovery, providing information, and mediating in conflicts with clinicians or family. In these cases, supplemental appointments with the conflicting parties were possible. All meetings and activities delivered were documented by the peer providers in a short protocol for each meeting.

\subsubsection{Organisation of Peer support}

Eight female and two male peer supporters were recruited to deliver services. Their mean age was 49.3 years (SD 6.6), ranging from 34 to 61 years. All of them had at least ten years of own experience with severe mental health problems, reflected their path to recovery, and 
found ways to handle upcoming crisis. Four peer supporters had previous experience in the role, six of them acted as peer supporters for the first time.

Peer supporters were associated with a local voluntary organisation for peer support (Ex-In), with a minimum of two peer supporters per participating hospital to allow for some organisational flexibility and peer-team intervision. They received limited reimbursement (i.e., so called "€450 per month jobs" or "mini-jobs" in accordance with German legislation). Peer supporters were allocated to patients according to organisational priorities. They contacted patients within the first week after randomisation and then established one-toone meetings. Patients were given the possibility to request a different peer supporter if they so wished.

The meetings took place in the offices of the peer supporters or in the community when patients needed support visiting a doctor, agency, or local authority. In a discussion group held prior to the trial where three peer supporters, one peer supervisor, and two researchers took part, the interventions modalities were shaped. We considered the minimum number of meetings required to build a supporting relationship and be effective for the patient, based on the experiences in delivering support by the peers themselves. Thus, preserving the necessary flexibility, a minimum number of four meetings in total, and a maximum frequency of one meeting per week was defined as appropriate. Meetings were supposed to last about one hour. Thus, patients and peer supporters were expected to meet between 4 and 26 times for an hour over a six-month period.

\subsubsection{Control condition and treatment as usual}

Both groups received their treatment as usual. This included in- and out-patient care with infrequent meetings with outpatient psychiatrists, and access to community based groups and separate psychological treatments. The control group did not receive any additional intervention beyond treatment as usual.

\subsection{Outcomes}

At baseline, socio-demographic characteristics were assessed. Clinical diagnoses according to ICD-10 were obtained from medical records.

\subsubsection{Primary outcome}

The primary outcome was self-efficacy, which was self-rated on the German version of the General Self-Efficacy Scale (GSE). The GSE has ten items, each rated on a four-point Likert scale from 1 (totally agree) to 4 (totally disagree), with higher scores indicating a higher level of self-efficacy. The GSE was developed by Schwarzer [22], based on Bandura's social cognitive theory. It is applicable for all diagnoses, and has been used in samples with cancer and mental disorders. Good internal consistency, with Cronbach's alpha between .76 - .91, and satisfactory validity have been reported [23].

\subsubsection{Secondary outcomes}

\subsubsection{Self ratings}

Subjective quality of life was measured with the Modular System for Quality of Life [24], a questionnaire specific for mental disorders, with seven dimensions on a seven-point Likert scale providing a total score between 1 and 7 , with higher scores reflecting better quality of life. Additionally, the EuroQol Questionnaire EQ-5D [25] was used, a widely established instrument with five items on different domains which build an index and a visual scale for the overall health status $(0-100)$ as a sixth domain. Higher scores indicate a higher level of quality of life.

\subsubsection{Clinician ratings}


The psychiatrist in charge of the treatment of the patient assessed the level of functioning of the patient on the Global Assessment of Functioning scale [26], with a single score between 1 and 100 with higher scores indicating better functioning. Severity of illness was measured with the Clinical Global Impression scale [27], with a single score seven-point scale (1-7), with a higher score indicating a higher severity of illness.

\subsubsection{Service use}

Whether patients were admitted to hospital within the study period and, if so, the number of days spent in hospital were taken from the medical records of the hospitals.

\subsection{Sample size}

A sample size of 158 patients ( 79 patients per group) was needed to detect a medium effect size (Cohen's $d=0.45$ ), i.e. a difference between groups of 2.4 points with a common standard deviation of 5.4 [22] on the self-efficacy scale (GSE), with a power of $80 \%$ and significance level of $5 \%$. Assuming a dropout rate of $20 \%$, a total sample of 198 patients (99 patients per group) was required.

\subsection{Statistical analysis}

Baseline characteristics are presented with descriptive statistics for demographic variables. For categorical variables, absolute and relative frequencies are provided. Continuous variables are presented by arithmetic means and standard deviations (SD).

The intention to treat analyses of primary data were based on the available clinical data from all randomised patients after the 12 month follow up. A mixed linear repeated measurement model (MMRM) was performed with patients as a random factor, the two follow-ups at 6 and 12 months' time points and group as fixed factors, and the baseline levels of the respective outcome as a covariate. We used the MMRM to model the dependency in the data set correctly, as these models are more robust against dropouts than models without random factor [28]. This analysis was performed for the primary and all secondary outcomes. The adjusted means and their differences between groups, as well as, the $95 \%$ confidence intervals $(95 \% \mathrm{Cl}), \mathrm{p}$-values and Cohen's $d$, are reported in tables and figures.

We also computed a per-protocol analysis, taking a minimum of four meetings between peer supporter and patient as the criterion for a per-protocol intervention.

Additionally, we conducted post-hoc analyses with diagnoses, gender, age, living situation, and education level as supplemental fixed effects to our primary model. In a second step, we conducted a backward selection of the not significant variables.

Throughout all analyses, the two-sided level of significance was set at $p<0.05$. Statistical analyses were carried out with SPSS, Version 20 [29].

This trial is registered at clinicaltrial.gov: NCT02276469 and received approval from the Ethics Committee of the State Chambers of Physicians Hamburg (Ref-Nr. PV3669).

\subsection{Role of the funding source}

The funding source had no influence in study design, namely, the collection, analysis, and interpretation of data; writing of the report; or the decision to submit the paper for publication.

\section{Results}

\subsection{Patient flow/Recruitment}

Between July 2011 and June 2013, 233 eligible individuals were identified of which 216 were recruited to the study; 114 (53\%) were allocated to the intervention of peer support and 102 $(47 \%)$ to the control group. At baseline, $87(40 \%)$ patients were in in-patient care, $46(53 \%)$ 
of them in the intervention, and $41(47 \%)$ in the control group. The patient flow throughout the study is shown in Figure 1.

Three patients changed the peer supporter after the first session. In two cases, the participants were unhappy with the original allocation and in one case, the peer supporter was unhappy with the allocation. In all three cases, the peer support continued after the change. The mean number of meetings between peer supporter and patients in the intervention group was 12.2 (SD 9.6) with a range of 0-40 sessions.

$$
\text { ===========Insert figure } 1 \text { approximately here============ }
$$

Whilst the dropout rate on the primary outcome was $31 \%$, it was substantially higher on the clinician-rated secondary outcomes. At the time of follow-ups, 109 (51\%) patients had been treated by psychiatrists in private office practice from whom outcomes could be obtained for only $46(21 \%)$ of patients. Forty patients (19\%) had no clinician at follow-up.

\subsection{Baseline data}

Patients' demographic and clinical characteristics at baseline are illustrated in table 1.

$$
==========\text { Insert table } 1 \text { approximately here============ }
$$

Overall, the patient sample included slightly more women 124 (56\%) than men, with a mean age of 41.48 years $(S D=12.28)$. Other characteristics and diagnoses were similar in both groups.

\subsection{Primary outcome}

Outcomes are shown in table 2 . Self-efficacy scores at six-month follow-up were significantly better in the intervention group, with an effect size of 0.3 .

==========-Insert table 2 approximately here============

\subsection{Secondary outcome}

There was no significant difference on patient or clinician rated secondary outcomes, as shown in table 3 . Sixty-two (54\%) patients in the intervention group and $50(49 \%)$ in the control group were admitted to hospital within the one-year study period. On average, the intervention group spent 24.9 days $(S D=41.6)$ and the control group 30.3 days $(S D=57.6)$ in hospital. The difference was not statistically significant, with an adjusted mean difference of 6.44 days $(95 \% \mathrm{Cl}-6.01-18.90, \mathrm{p}=0.31)$.

===========Insert table 3 approximately here============

\subsection{Per-protocol analyses}

The per-protocol analyses are indicated in table 2. Following the intervention as planned, results on self-efficacy showed a larger and significant difference with an effect size of $d=0.4$. In the per-protocol analyses, the only statistically significant difference was found for the VAS of the EQ 5D, which suggested a more positive quality of life in the intervention group (95\% Cl 0.82-10.49, $p=0.04)$.

\subsection{Post-hoc analyses}

The post hoc analyses showed that no significant effect of participants' diagnoses, gender, age, living situation, or education level could be detected. 


\subsection{Harms}

One participant died of a natural cause during the follow-up period.

\section{Discussion}

\subsection{Main findings}

Participants receiving one-to-one peer support rated their self-efficacy significantly higher during a follow-up period of six months after treatment. There was no statistically significant benefit in secondary outcomes. Further analyses with a per-protocol analysis revealed largely similar findings.

\subsection{Strengths and limitations}

The trial had several strengths considering the existing literature on the effectiveness of peer support. The role of the peer supporters was clearly set out and in line with what has been suggested as best practice. The peer supporters were extensively trained in their role. The trial was sufficiently powered for the primary outcome, and the primary outcome of selfefficacy was recovery-oriented and directly linked to the objectives and tasks of the one-to one peer support. Moreover, the method of obtaining the primary outcome through postal questionnaires excluded any interviewer bias, although it might also have led to a lower response rate than might have been achieved in personal interviews.

However, there are also some limitations. In particular, the low rate of clinician ratings, with a dropout rate of $67 \%$ in the control group, makes the results of clinical outcomes very difficult to interpret. Consequently, there is a lack of detailed assessments of patient experiences. However, they would have required personal interviews in which it might not have been feasible to maintain the blinding of interviewers. Systematically collected data on further services patients accessed over time are also missing. Further research, whether peer support is bridging and engages service users with mental health services is indicated. Finally, independently of poor response rates, the study was not powered to detect differences in secondary outcomes although it is important to know whether improvements in self-efficacy translate in better quality of life and lower symptom levels.

\subsection{Comparison with findings from published studies/reviews}

Improved self-efficacy is linked to the concept of personal recovery. People with higher selfefficacy have been shown to accept more challenging tasks, persist longer at tasks, and overcome setbacks more quickly [30]. Higher self-efficacy predicts more appropriate coping strategies, acceptance of life imperfection, and personal growth [12], whilst lower selfefficacy can be associated with self-stigmatising beliefs and lack of empowerment [31].

Only two previous trials on peer support used self-efficacy as an outcome. They tested peer delivered groups for patients with schizophrenia and compared them with a waiting group on peer support. One trial did not demonstrate an effect of peer support, and the other one identified a positive effect after six months, albeit with a smaller effect size than found in our study $[32,33]$. Our findings go beyond that and show that a one-to-one peer support can lead to improved self-efficacy with a larger effect size than hitherto suggested and over a one-year period. Thus, the results affirm Gillards` change model.

The absence of significant effects on hospital admissions is in line with other previous trials. Other trials suggested a positive effect on quality of life, which we failed to identify in the intention to treat analyses, but with inconsistent findings regarding the per-protocol analyses. 


\subsection{Clinical and research implications}

The wide implementation of peer support may be seen as an important component of a recovery oriented and destigmatising service with a culture of collaboration across professionals, patients, and carers [1]. This collaboration has also been termed 'trialogue' and promoted through a wide range of initiatives, originating in German-speaking countries $[34,35]$. Such effects on the culture of organisations may be difficult to establish in experimental research, particularly as they are likely to take time before having a full impact. However, building on other trials on peer support, this study shows that one-to-one peer support can also have direct benefits for participants with severe illnesses and improve their self-efficacy, independent from the diagnosis or sociodemographic characteristics, like living situation or education. Further research and possibly trials with larger samples are required to explore how this improved self-efficacy can translate into clinical and social gains in patients with severe mental illnesses. Programmes for peer support are developing, and may be improved in the future through a better selection of participants, a more precise definition of the role of the peer, and more flexible arrangements for how and over what periods of time support will be provided.

The findings of this trial suggest that a systematic training of peers and a preparation of staff in participating services may be an essential ingredient in effective programmes.

\section{Declaration of interest}

None of the authors had competing interest.

\section{Contributors}

$\mathrm{CM}, \mathrm{SP}, \mathrm{KW}$ and TB conceived of the research question and/or designed the study. $\mathrm{CM}, \mathrm{KH}$ and $T B$ oversaw the data acquisition. $A D$ and $K W$ oversaw the analysis. $C M, K H$ and $A D$ analysed the data. $\mathrm{CM}, \mathrm{SP}, \mathrm{KH}$ and TB interpreted the data. $\mathrm{CM}, \mathrm{SP}, \mathrm{AD}$ and $\mathrm{KH}$ drafted the report. All authors provided critical revisions to the report, important intellectual content, and final approval.

\section{Acknowledgment}

This project was part of the 'psychenet' project (www.psychenet.de) and received funding from the Federal Ministry of Education and Research in Germany from 2011 until 2015 by, (BMBF-Nr: O1KQ1002B).

We thank all our peer workers for their great effort. In addition, we thank Tuula Rouhiainen from the user organisation for individuals with psychiatric experience, Hans Jochim Meyer from the organisation of relatives of individuals with psychological illness, Prof. Dr. Martin Lambert, and Gyöngyver Sielaff from Ex-In, for their great project leadership. We also want to thank all clinical directors of the participating centres, who were so satisfied with the peer support that they decided to provide and finance it regularly. Finally, yet importantly, we would like to thank all participants of the trial.

\section{References}

[1] Slade M, Amering M, Farkas M, Hamilton B, O'Hagan M, Panther G, Perkins R, Shepherd $G$, Tse $S$, Whitley R. Uses and abuses of recovery: implementing recovery-oriented practices in mental health systems. World Psychiatry. 2014; 13: 12-20.

[2] Stierlin AS, Herder K, Helmbrecht MJ, Prinz S, Walendzik J, Holzmann M, Becker T, Schützwohl M, Kilian R. Effectiveness and efficiency of integrated mental health care programmes in Germany: study protocol of an observational controlled trial. BMC Psychiatry. 2014; 14: 163.

[3] Priebe S, Omer S, Giacco D, Slade M. Resource-oriented therapeutic models in psychiatry: a conceptual review. B J Psych. 2014; 204: 256-61. 
[4] Faulkner A, Basset T. A long and honourable history. The Journal of Mental Health Training, Education and Practice. 2012; 7: 53-59.

[5] Wallcraft J, Amering M, Freidin J. Partnerships for better mental health worldwide: WPA recommendations on best practices in working with service users and family carers. World Psychiatry. 2011; 10: 229-36.

[6] Haro JM, Ayuso-Mateos JL, Bitter I, Demotes-Mainard J, Leboyer M, Lewis SW, Linszen D, Maj M, McDaid D, Meyer-Lindenberg A, Robbins TW, Schumann G, Thornicroft G, Van Der Feltz-Cornelis C, Van Os J, Wahlbeck K, Wittchen HU, Wykes T, Arango C, Bickenbach J, Brunn M, Cammarata P, Chevreul K, Evans-Lacko S, Finocchiaro C, Fiorillo A, Forsman AK, Hazo JB, Knappe S, Kuepper R, Luciano M, Miret M, Obradors-Tarragó C, Pagano G, Papp S, Walker-Tilley T. ROAMER: a European roadmap for mental health research. Int J Meth Psychiatry Res 2014; 23(Suppl 1): 1-13.

[7] Stamou E. Reclaiming user leadership in peer support practice. J MH Training, Ed and Practice. 2014; 9: 167-76.

[8] Chinman M, George P, Dougherty R, Daniels A, Ghose S, Swift A, Delphin-Rittmon ME. Peer support services for individuals with serious mental illnesses: assessing the evidence. Psychiatric Services. 2014; 65:429-41.

[9] Mahlke C, Krämer U, Becker T, Bock T. Peer support in mental health services. Current opinion in psychiatry. 2014; 27: 276-81.

[10]Mead S, Hilton D, Curtis L. Peer support: A theoretical perspective. Psychiatr Rehabil J 2001; 25:134-141.

[11] Gillard S, Gibson S L, Holley J, Lucock M. Developing a change model for peer worker interventions in mental health services: a qualitative research study. Epidemiology and Psychiatric Science: 2014; 1-11

[12] Luszczynska A, Scholz U, Schwarzer R. The General Self-Efficacy Scale: Multicultural Validation Studies. J Psychol. 2005; 139: 439-57.

[13]Fuhr DC, Salisbury TT, De Silva MJ, Atif N, van Ginneken N, Rahman A, Patel V. Effectiveness of peer-delivered interventions for severe mental illness and depression on clinical and psychosocial outcomes: a systematic review and meta-analysis. Soc Psychiatr Epidemiol. 2014; 49: 1691-702.

[14]Pitt V, Lowe D, Hill S, Prictor M, Hetrick SE, Ryan R, Berends L. Consumer-providers of care for adult clients of statutory mental health services. Cochrane Database of Systematic Reviews. 2013; 3.

[15] Lloyd-Evans B, Mayo- Wilson E, Harrison B, et al. A systematic review and meta-analysis of randomized controlled trials of peer support for people with severe mental illness. BMC Psychiatry. 2014; 39; 1-12.

[16] Doughty C, Tse S. Can consumer-led mental health services be equally effective? An integrative review of CLMH services in high-income countries. Community Ment Health J 2011; 47: 252-66.

[17]Gühne U, Weinmann S, Arnold K, Becker T \& Riedel-Heller SG. S3 guideline on psychosocial therapies in severe mental illness: evidence and recommendations. Eur Arch Psychiatry Clin Neurosci. 2015; 265:173-188.

[18] Repper J. Peer Support Workers: a practical guide to implementation. Implementing Recovery through Organisational Change, Briefing Paper. 2013; 7, http://www.imroc.org/media/publications.

[19]Moran G, Russinova Z, Gidugu V, Gagne C. Challenges Experienced by Paid Peer Providers in Mental Health Recovery. Community Ment Health J. 2013; 49:281-291.

[20] Kemp V, Henderson AR. Challenges faced by mental health peer support workers: peer support from the peer supporter's point of view. Psychiatr Rehabil J. 2012; 35:337-340.

[21] Utschakowski J. Training programme for people with experience in mental health crisis to work as trainer and peer supporter. Ex-In Curriculum; 2007; Available at: 
http://www.adam-europe.eu/prj/1871/prd/3/1/Curriculum_english\%20i.pdf last: 11.09.2016.

[22]Schwarzer R, Jerusalem M. Generalized Self-Efficacy scale. In: Weinman J, Wright S, Johnston $\mathrm{M}$, eds. Measures in health psychology: A user's portfolio. Causal and control beliefs. Windsor, UK: NFER-NELSON, 1995: 35-37.

[23]Schwarzer, R. General Perceived Self-Efficacy in 14 cultures [online-publication]. Berlin: Freie Universität Berlin 2001. Available at: http://userpage.fu berlin.de/ health/world14.htm. last: 18.05.2016.

[24]Pukrop R. Modulares System zur Lebensqualität. In Schumacher J, Klaiberg A, Brähler E (Hrsg.), Diagnostische Verfahren zu Lebensqualität und Wohlbefinden. Göttingen Bern Toronto S; 2003; 227-232.

[25]EuroQol Group. EuroQol-a new facility for the measurement of health-related quality of life. Health Policy. 1990; 16:199-208.

[26] Sass H, Wittchen HU, Zaudig M. Diagnostisches und Statistisches Manual psychischer Störungen DSM-IV. Göttingen, Bern, Toronto, Seattle: Hogrefe; 2001.

[27]National Institute of Mental Health. Clinical Global Impressions. In: Guy W, Ed. ECDEU Assessment for psychopharmacology. Rev. Ed. Rockville, Maryland; 1976; 217-222.

[28] Brown H, Prescott R: Repeated measures data. In: Applied Mixed Models in Medicine. New York, NY: John Wiley \& Sons: 2015: 231-288.

[29]IBM Corp. IBM SPSS Statistics for Windows, Version 20.0. Armonk, NY: IBM Corp, 2011.

[30]Schwarzer R, Bäßler J, Kwiatek P, Schröder K, Zhang JX,. The assessment of optimistic selfbeliefs: comparison of the German, Spanish, and Chinese versions of the General SelfEfficacy Scale. Applied Psychology: An International Review. 1997; 46: 69-88.

[31] Vauth R, Kleim B, Wirtz M, Corrigan PW. Self-efficacy and empowerment as outcomes of self-stigmatizing and coping in schizophrenia. Psyciatr Research. 2007; 150: 71-80.

[32] Castelein S, Bruggeman R, van Busschbach J T, van der Gaag M, Stant A D, Knegtering $H$ et al. The effectiveness of peer support groups in psychosis: A randomized controlled trial. Acta Psychiatrica Scandinavica. 2008; 118: 64-72.

[33]van Gestel-Timmermans H, Brouwers EPM, van Assen M, van Nieuwenhuizen C. Effects of a peer-run course on recovery from serious mental illness: a randomized controlled trial. Psychiatr Serv. 2012; 63: 54-60.

[34]Bock T, Priebe S. Psychosis-seminars, an unconventional approach for how users, carers and professionals can learn from each other. Psychiatr Serv. 2005; 56: 1441-1443.

[35]Amering M, Mikus M, Steffen S. Recovery in Austria: mental health trialogue. Int Rev Psychiatry. 2012; 24: 11-8. 